Research Article

\title{
The Interaction between Bmal1 and Per2 in Mouse BMSC Osteogenic Differentiation
}

\author{
Haiya Zhuo $\mathbb{D}^{\text {, }}$, Yuhong Wang $\mathbb{D}$, and Qing Zhao $\mathbb{1}$ \\ State Key Laboratory of Oral Diseases, Department of Orthodontics, West China Hospital of Stomatology, Sichuan University, \\ Chengdu, China \\ Correspondence should be addressed to Qing Zhao; fanfan_qing@163.com
}

Received 2 October 2017; Accepted 11 February 2018; Published 29 March 2018

Academic Editor: Dominique Bonnet

Copyright (c) 2018 Haiya Zhuo et al. This is an open access article distributed under the Creative Commons Attribution License, which permits unrestricted use, distribution, and reproduction in any medium, provided the original work is properly cited.

\begin{abstract}
The circadian clock is a system that controls endogenous time of organisms, and it regulates the physiology and behavior of bodies. The transcription factors Brain and Muscle ARNT-like Protein 1 (BMAL1) and Period2 (Per2) are components of the circadian clock, and they play vital roles in circadian clock function. Both Bmal1-/- mice and Per2-/- mice display obvious bone volume changes. In this study, we inhibited the expression of Bmall in bone marrow-derived mesenchymal stem cells (BMSCs) using a lentiviral vector harboring RNAi sequences, which increased the osteogenic differentiation capability of BMSCs. We also suppressed Per2 gene expression using an adenovirus vector harboring RNAi sequences, and similarly, the osteogenic differentiation ability of BMSCs was enhanced. Furthermore, when both Bmal1 and Per2 gene expression was suppressed in BMSCs by lentiviral and adenoviral interference, the osteogenic differentiation capability was stronger than that in BMSCs following single-gene inhibition. Our data support that both Bmal1 and Per2 play negative roles in BMSC osteogenic differentiation and that Bmall and Per2 have a synergistic effect on the osteogenic differentiation of BMSCs.
\end{abstract}

\section{Introduction}

Bone is one of the most active organs in organisms, and it remodels throughout life. Research shows that there are some signaling pathways involved in bone metabolism, and the circadian clock is among them [1]. Serious consequences may appear if the circadian rhythm has disordered, such as a decrease of the bone mineral density as well as an increased risk of patients developing osteoporosis $[2,3]$. Genes that take part in mesenchymal stem cell and osteoblast differentiation and genes that participate in mineral deposition are all expressed rhythmically [4-6]. Bmal1 and Per2 are both important components of the molecular biological clock, and they are expressed in bone with 24-h periodicity [7]. Mice that are deficient in Bmall show an accelerated aging phenotype and have no orthotropic osteogenesis, and older mice that are deficient in Bmall have lower bone weights $[8,9]$. Bmal1-/- mice occur with low bone mass [10], but another study showed that Bmal1-/- mice had elevated osteoblasts numbers and bone-formation parameters (MAR: mineral apposition rate; BFR: bone-formation rate), indicating that Bmall inhibits bone formation. Studies demonstrate that Per1-/-; Per2m/m, Per1-/-; Per2-/-, and Per $2^{\mathrm{Brdm} 1}$ mutant mice all exhibit high bone mass phenotypes $[11,12]$. Thus, Bmall and Per2 play vital roles in the regulation of bone formation. Additionally, Bmal1 and Per2 are both the core components of the circadian clock autoregulatory feedback loop. Whether Bmal1 and Per2 have an interaction in the regulation of bone formation is still unknown.

To explore the specific roles of Bmall and Per2 in the regulation of bone formation and to determine whether Bmal1 and Per2 have an interaction in this process, we inhibited the expression of Bmal1 or/and Per2 in BMSCs and observed their ability to differentiate into osteoblasts. The data showed that the mRNA and protein expression of osteogenic differentiation markers (Alp, Runx2, and Ocn), the ALP activity, and the ability of calcified nodule formation of BMSCs were enhanced after Bmall inhibition, which indicated that Bmall has a negative role in the regulation of osteogenic differentiation in BMSCs. After the inhibition of Per2, the osteogenic differentiation capability of BMSCs was similarly enhanced, demonstrating that Per2 also has a negative role in the 
regulation of osteogenic differentiation in BMSCs. After the simultaneous suppression of Bmal1 and Per2, the osteogenic differentiation capability of BMSCs were much stronger than those in BMSCs following single-gene inhibition, which indicated that Bmal1 and Per2 have a synergistic effect on the osteogenic differentiation of BMSCs.

\section{Materials and Methods}

2.1. Cell Culture. BMSCs (passage 5) isolated from 4-weekold C57/BL6 male mice were purchased from the Cyagen Biosciences incorporated company (Guangzhou, China). The BMSCs were identified as CD29(+), CD44(+), CD117(-), and CD31(-), and passage 12 BMSCs had good adipogenesis and osteogenesis abilities, as described by the seller. The cells were cultured in a humidified atmosphere with $5 \% \mathrm{CO} 2$ at $37^{\circ} \mathrm{C}$ and fresh alpha-minimum essential medium $(\alpha$-MEM) containing $10 \%$ fetal bovine serum (FBS), $100 \mathrm{mg} / \mathrm{ml}$ streptomycin, and $100 \mathrm{U} / \mathrm{ml}$ penicillin (basic medium); the medium was changed 2-3 times per week. When BMSCs were $80 \%-90 \%$ confluent, they were passaged using $0.25 \%$ trypsin and $0.01 \%$ ethylenediaminetetra acetic acid (EDTA). The passage 5 cells were used in the experiments below.

2.2. Experimental Groups. There were seven groups in the present study: the vacant control BMSC group (group 1, Vacant), the Bmall shRNA lentivirus vector-transfected BMSC group (group 2, Bmal1 repression group, LentiBmall shRNA), the green fluorescent protein- (GFP-) expressing lentivirus vector-transfected BMSC group (group 3, Lenti-GFP-Puro), the Per2 shRNA adenovirus vectortransfected BMSC group (group 4, Per2 repression group, Adeno-Per2 shRNA), the red fluorescent protein- (RFP-) expressing adenovirus vector-transfected BMSC group (group 5, Adeno-RFP-Puro), the Bmall shRNA lentivirusand Per2 shRNA adenovirus-transfected BMSC group (group 6, Bmal1 and Per2 repression group, Lenti-Bmal1 shRNA-Adeno-Per2 shRNA), and the GFP-expressing lentivirus and RFP-expressing adenovirus-transfected BMSC group (group 7, Lenti-GFP-Puro-Adeno-RFP-Puro). Regarding the last two groups, we first constructed stable Bmal1 shRNA lentivirus-transfected BMSC lines and GFP-expressing lentivirus-transfected BMSC lines and then added Per2 shRNA and RFP-expressing adenovirus, respectively.

2.3. The Construction of Stable Bmal1 shRNA-Transfected BMSC Lines and GFP-Expressing Lentivirus-Transfected BMSC Lines. The passage 5 BMSCs were seeded in 6-well plastic plates at an initial density of $5 \times 10^{5}$ cells per well in basic media, and the Bmall shRNA lentivirus (Arntl shRNA, synthesized by Hanheng bio, Shanghai, China) (group 2, Bmal1 repression group, Lenti-Bmal1 shRNA) or the GPFexpressing lentivirus (pHBLV-U6-ZsGreen-Puro) (group 3, Lenti-GFP-Puro) was added per well at a multiplicity of infection (MOI) of 20 . After $24 \mathrm{~h}$ of cell culture, the medium was replaced with the basic one, and 48-h later, the cells were screened by basic medium supplemented with $2 \mu \mathrm{g} / \mathrm{ml}$ puromycin. The green fluorescence level was observed under an inverted fluorescence microscope (OLYMPUS IX70, Japan), and we calculated the transfection efficiency by RT-qPCR and Western blotting assays. The cells were passaged until they were stable, and after two passages, the adequate cells were cryopreserved for the following experiments.

2.4. Per2 shRNA Adenovirus Vector and RPF-Expressing Adenovirus Vector Transfection. The BMSCs, stable Bmal1 shRNA-transfected BMSCs and GFP-expressing lentivirustransfected BMSC, were cultured in 24-well plastic plates at an initial density of $4 \times 10^{4}$ cells per well in basic media, and after the cells were $80 \%$ confluent, the Per 2 shRNA adenovirus vector (synthesized by Hanheng bio, Shanghai, China) was added to the wells containing BMSCs and Bmal1 shRNA-transfected BMSCs (group 4, Adeno-Per2 shRNA and group 6, Lenti-Bmal1 shRNA-Adeno-Per2 shRNA, resp.), and the RFP-expressing adenovirus vector (synthesized by Hanheng bio, Shanghai, China) was added to the wells containing BMSCs and GFP-expressing lentivirustransfected BMSC (group 5, Adeno-RFP-Puro and group 7, Lenti-GFP-Puro-Adeno-RFP-Puro, resp.) at an MOI of 20. The fluorescence levels were observed under an inverted fluorescence microscope after $48 \mathrm{~h}$, and we determined the transfection efficiency using Western blotting assays. The dilution of anti-BMAL1 (1:500), anti-PER2 (1:500) and anti- $\beta$-actin primary antibodies $(1: 1000)$ were used. And the dilution of horseradish peroxidase-conjugated secondary antibody $(1: 1000)$ was used.

2.5. RT-qPCR Analysis. The cells in those seven groups were cultured in 6-well plates with $1 \times 10^{5}$ cells per well, and several groups were transfected by the corresponding adenovirus as required. After the cells were $80 \%$ confluent, they were cultured in the osteogenic medium (basic medium together with $50 \mu \mathrm{g} / \mathrm{ml} \mathrm{L}$-ascorbate, $10 \mathrm{nM}$ dexamethasone, and $10 \mathrm{mM} \beta$-glycerophosphate), and mRNA expression was evaluated after 7 and 14 days of osteoinduction. The cells were harvested and kept in an RNA preservation solution (RNA safeguard). The total RNA was extracted applying a simple P total RNA extraction kit. The absorbance (A) at $260 \mathrm{~nm}$ was measured using a spectrophotometer to quantify the total RNA, and an A260: A280 ratio of 2.0 of RNA samples was considered to be of high purity. Two milligrams of total RNA acquired from each sample was used for reverse transcription applying the SYBR-Prime ScriptTM RT-PCR Kit following the manufacturer's protocol. The real-time PCR was carried out in a $20-\mu$ reaction mixture in triplicate on an ABI PRISM 7300 Real-time PCR system (Applied Biosystems, USA) according to the manufacturer's protocol. The housekeeping gene $\beta$-actin was used as the internal reference, and primers applied to real-time PCR analysis are shown in Table 1 . We calculated the initial copy numbers of unknown samples using the 7300 Real-time PCR system from the standard curve.

2.6. Western Blotting. The cells in those seven groups were cultured in 6-well plates with $1 \times 10^{5}$ cells per well, and some groups were transfected by the corresponding adenovirus as required. After cells were $80 \%$ confluent, they were cultured 
TABLE 1: The primers used for RT-qPCR analysis. ALP: alkaline phosphatase; Runx2: runt-related transcription factor 2; and Ocn: osteocalcin.

\begin{tabular}{|c|c|c|c|}
\hline Target gene & Primers & Sequence & Fragment size (bp) \\
\hline \multirow{2}{*}{ ALP } & Forward primer & $5^{\prime}$ - ATAACGAGATGCCACCAGAGG-3' & \multirow{2}{*}{$140 \mathrm{bp}$} \\
\hline & Reverse primer & 5' - TTCCACATCAGTTCTGTTCTTCG-3' & \\
\hline \multirow{2}{*}{ RUNX2 } & Forward primer & $5^{\prime}$ - CTACCCAGCCACCTTTACCTAC-3' & \multirow{2}{*}{$190 \mathrm{bp}$} \\
\hline & Reverse primer & $5^{\prime}$ - GAACTGATAGGATGCTGACGAAG-3' & \\
\hline \multirow{2}{*}{ OCN } & Forward primer & $5^{\prime}$ - AGGAGGGCAATAAGGTAGTGAAC-3' & \multirow{2}{*}{$147 \mathrm{bp}$} \\
\hline & Reverse primer & 5' - AGGCGGTCTTCAAGCCATAC-3’ & \\
\hline \multirow{2}{*}{$\beta$-actin } & Forward primer & 5' - GAGACCTTCAACACCCCAGC-3' & \multirow{2}{*}{$263 \mathrm{bp}$} \\
\hline & Reverse primer & 5' - ATGTCACGCACGATTTCCC-3' & \\
\hline
\end{tabular}

in the osteogenic medium as described in the RT-qPCR protocol, and total protein was obtained after 7 and 14 days. GAPDH (Glyceraldehyde-3-phosphate dehydrogenase) or $\beta$-actin were used as internal references. After being cultured for the abovementioned times, those cells were washed with ice-cold phosphate-buffered saline (PBS) twice and then lysed by lysis buffer from the Keygen total protein extraction kit. Then we collected the supernatant after the lysate was centrifuged at $14,000 \mathrm{~g}$ for $15 \mathrm{~min}$ at $4^{\circ} \mathrm{C}$ and the supernatant was quantitatively assayed using the BCA (bicinchoninic acid) method. We disposed total protein extracts using standard SDS-PAGE procedures and subsequently transferred them to a PVDF membrane. And after blocking, we probed the membranes using the appropriate specific primary antibodies and horseradish peroxidase- (HRP-) conjugated secondary antibodies. We observed the immunoreactive proteins applying a chemiluminescence kit. The ChemiDoc XRS Gel documentation system and Quantity One software (Bio-Rad) were used to determine the band intensities.

2.7. Quantitative Alkaline Phosphatase and Protein Assays. The cells in those seven groups were cultured in 6-well plates with $1 \times 10^{5}$ cells per well, and some groups were transfected by the corresponding adenovirus as required. To quantitatively analyze alkaline phosphatase activity, PBS was used to wash the cells after osteoinduction for 7 days, and $10 \mathrm{mM}$ Tris $\mathrm{HCl}$ and $0.1 \%$ Triton $\mathrm{X}-100$ (pH 7.4) were used to lyse the cells. These cells were then processed by repeating the freeze-thaw cycle three times. After sonication, ALP activity was biochemically measured with a modified method of King applying a commercial kit as instructed. The total protein content was measured using the Bradford method; the reactions were evaluated at $510 \mathrm{~nm}$ and calculated based on bovine $\gamma$ globulin standards. The ALP data are expressed as unit/L protein.

2.8. Alizarin Red Staining. The cells in those seven groups were cultured in 24 -well plates with $4 \times 104$ cells per well, and some groups were transfected by the corresponding adenovirus as required. After cells were $80 \%$ confluent, they were cultured in the osteogenic medium as described in the RTqPCR protocol. After 14 days of osteogenic induction, 4\% paraformaldehyde was used to fix the cover glass from the experimental and control groups in 24-well plates for $20 \mathrm{~min}$. After the cells were rinsed with PBS, they were stained with Alizarin red staining solution and incubated at room temperature for $30 \mathrm{~min}$, and then the cells were observed under inverted phase microscope. The intensity of Alizarin red of each group was quantified by using the Image Pro-Plus 6.0.

2.9. Statistical Analysis. All experiments were conducted at least three times. Measurements are presented as the mean \pm SD. Statistical comparisons were determined by factorial analysis of variance (ANOVA), followed by the StudentNewman-Keuls (SNK) test to do the multiple comparisons. Statistical significances were considered at a value of $p<0.05$.

\section{Results}

3.1. Repression of Bmall and/or Per2 in $m R N A$ and Protein Levels after Virus Transfection in BMSCs. After $48 \mathrm{~h}$ of lentiviral infection, the inverted fluorescence microscope was used to detect the GFP in BMSCs (Figure 1(a)). As shown in Figure 1(b), the transfection efficiency was greater than $60 \%$, and the suppression of Bmall mRNA transcripts was successful in the Bmal1 repression group. Inhibition of the Bmall gene was further confirmed at the protein level by Western blot analysis (Figures $1(\mathrm{f})$ and $1(\mathrm{~g})$ ). The expression of the BMAL1 protein in the Bmal1 repression group displayed an obvious decrease compared with the Lenti-GFPPuro group. In addition, the expression of PER2 protein also decreased in the Bmall repression group.

After $48 \mathrm{~h}$ of adenoviral infection, RFP was detected in BMSCs under an inverted fluorescence microscope (Figure 1(c)). As shown in Figure 1(d), the transfection efficiency was greater than $70 \%$, and suppression of the Per2 mRNA transcripts was successful in the Per2 repression group. Inhibition of the Per2 gene was further confirmed at the protein level by Western blot analysis (Figures $1(\mathrm{f})$ and $1(\mathrm{~h})$ ). In addition, the expression of BMAL1 protein also decreased in the Per2 repression group.

After $48 \mathrm{~h}$ of lentiviral and adenoviral infection, GFP and RFP were detected in BMSCs under an inverted fluorescence microscope (Figure 1(e)). The Bmall shRNA lentivirus and Per2 shRNA adenovirus (in group 6) weakened the 

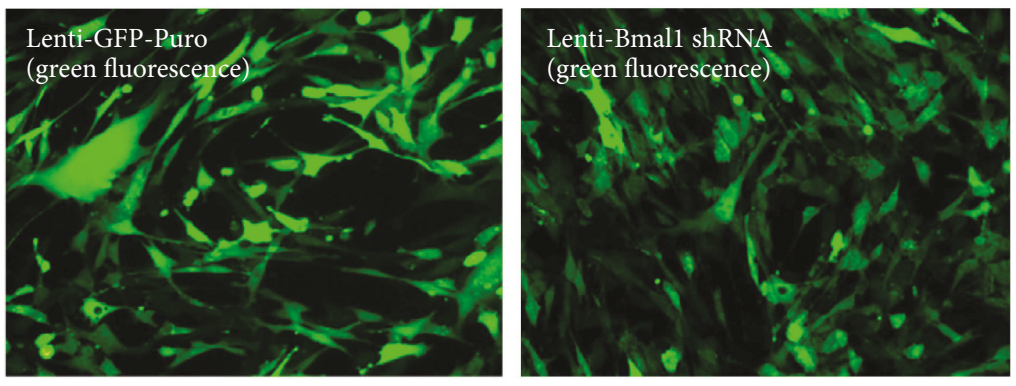

(a)
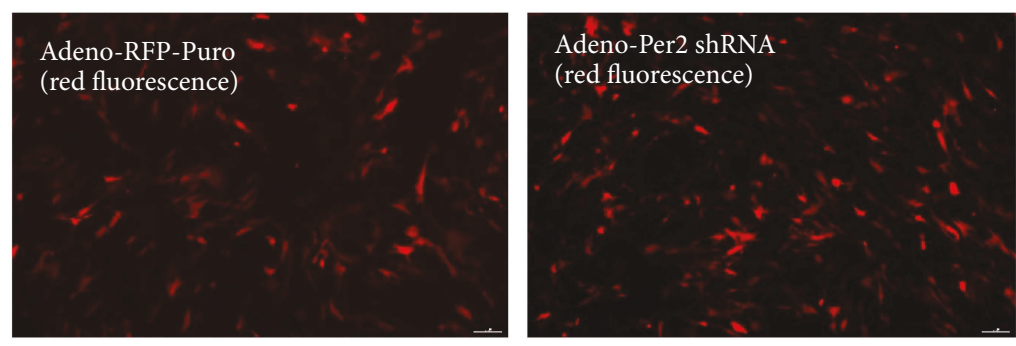

(c)
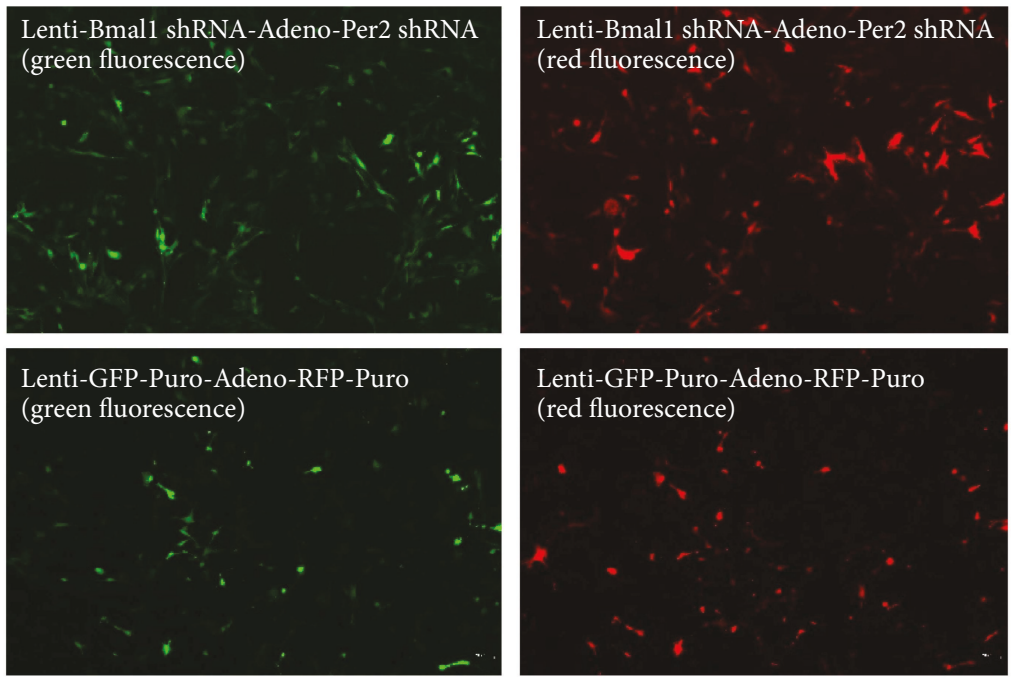

(e)

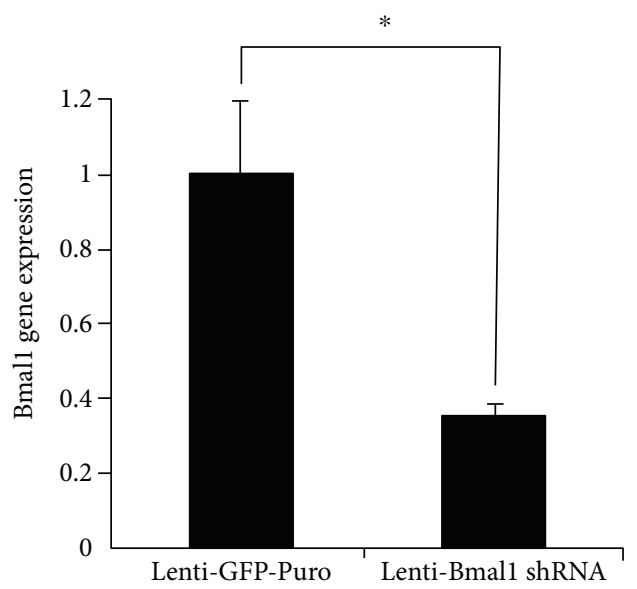

(b)

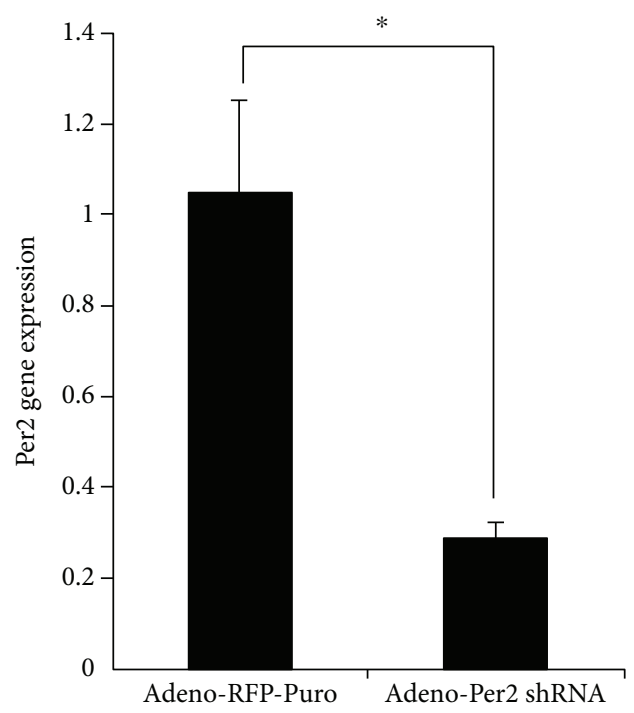

(d)

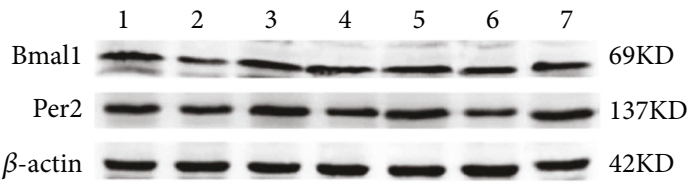

(f)

FIgURe 1: Continued. 


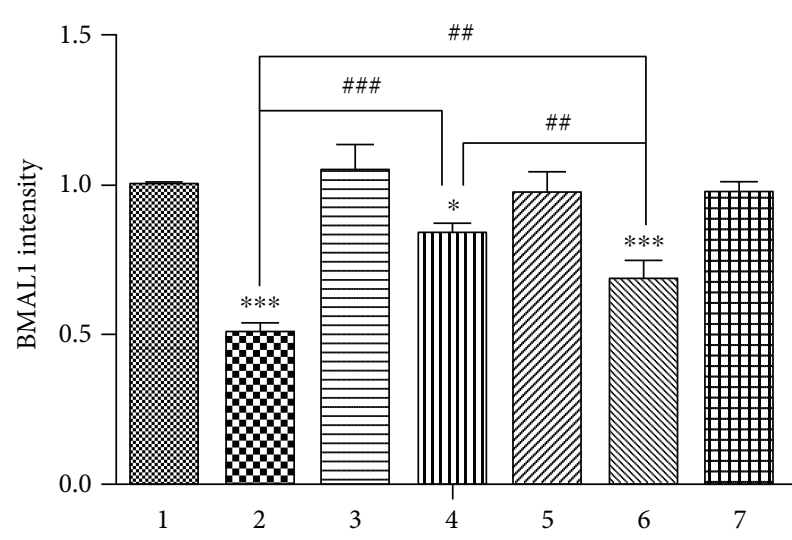

(g)

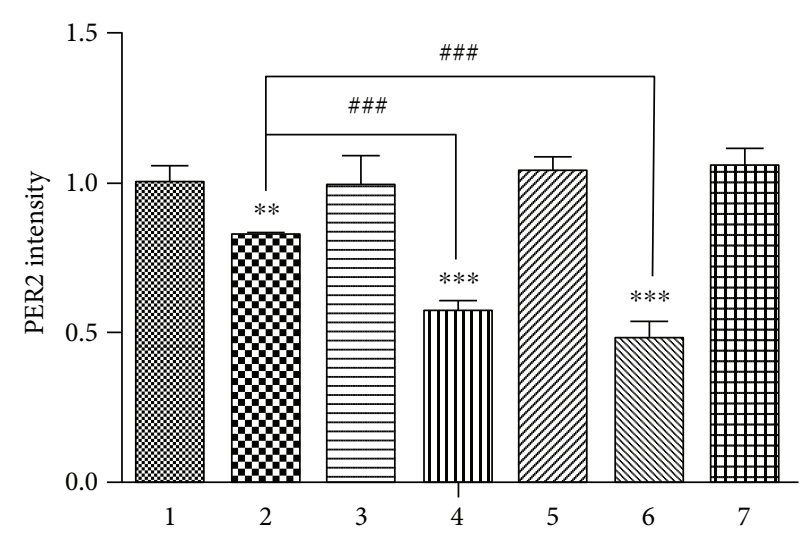

(h)

FIGURE 1: The decreased expression of Bmal1 and/or Per2 in mRNA and protein levels after virus transfection in BMSCs. (a) Expression of GFP in BMSCs infected by GFP-expressing lentivirus and Bmall shRNA lentivirus. After the BMSCs were infected for $48 \mathrm{~h}$, we can observe abundant GFP. Magnification is 200x. (b) The expression level of Bmal1 mRNA $48 \mathrm{~h}$ after transfection. (c) Expression of RFP in BMSCs infected by RFP-expressing adenovirus and Per2 shRNA adenovirus. After the BMSCs were infected for $48 \mathrm{~h}$, we can observe abundant expression of RFP. Magnification is 100x. (d) The expression level of Per2 mRNA $48 \mathrm{~h}$ after transfection. (e) Expression of GFP and RFP in the Bmal1 shRNA lentivirus- and Per2 shRNA adenovirus-transfected BMSC group (group 6, Lenti-Bmal1 shRNA-Adeno-Per2 shRNA) and the GFP-expressing lentivirus- and RFP-expressing adenovirus-transfected BMSC group (group 7, Lenti-GFP-Puro- AdenoRFP-Puro). After the BMSCs were infected for $48 \mathrm{~h}$, we can observe abundant expression of GFP and RPF. Magnification is 100x. (f-h) The expression level of BMAL1 and PER2 protein of BMSCs in each group $48 \mathrm{~h}$ after transfection. 1, 2, 3, 4, 5, 6, and 7 represented the Vacant, Lenti-Bmal1 shRNA, Lenti-GFP-Puro, Adeno-Per2 shRNA, Adeno-RFP-Puro, Lenti-Bmal1 shRNA-Adeno-Per2 shRNA, and Lenti-GFP-Puro-Adeno-RFP-Puro groups, respectively. The Bmal1 shRNA lentivirus (in group 2) weakened the expression of BMAL1 protein compared with the GFP-expressing lentivirus (in group 3), and it also downregulated the expression of PER2. The Per2 shRNA adenovirus (in group 4) weakened the expression of PER2 protein compared with the RFP-expressing adenovirus (in group 5), and it also downregulated the expression of BMAL1. In addition, the Bmal1 shRNA lentivirus and Per2 shRNA adenovirus (in group 6) weakened the expression of BMAL1 and PER2 protein compared with the GFP-expressing lentivirus and RFP-expressing adenovirus (in group 7). $\beta$ actin served as the endogenous reference gene. Densitometry by Quantity One software was used to quantify the protein level, and the protein level was displayed as the ratio of BMAL1 and PER2 relative to $\beta$-actin. Data represent the mean \pm SD $(n=3)$; the asterisk indicates the group compared to the control groups, that is, group 1, group 3, group 5, or group $7 .{ }^{*} p<0.05,{ }^{* *} p<0.01$, and ${ }^{* * *} p<0.001$. The pound key indicates a significant difference between the two groups $\left({ }^{\#} p<0.01,{ }^{\# \#} p<0.001\right)$.

expression of BMAL1 and PER2 protein compared with the GFP-expressing lentivirus and RFP-expressing adenovirus (in group 7) (Figures 1(f)-1(h)). Inhibition of Bmall and Per2 gene expression in all groups was confirmed at the protein level by Western blot analysis (Figures $1(\mathrm{f})-1(\mathrm{~h})$ ).

\subsection{Expression of Osteogenic Differentiation Markers in Each} Group after Osteoinduction. After 7 days of osteoinduction, there was no statistically significant difference in the mRNA expression of Alp, Runx2, and Ocn among group 1, group 3 , group 5, and group 7 (Figure 2), indicating that GFP and/or RFP does not interfere with the expression of Alp, Runx2, and Ocn in the four vacant virus groups. Inhibition of the Bmal1 or Per2 genes (in group 2 and group 4) enhanced the mRNA expression of Alp, Runx2, and Ocn (Figures 2(a)-2(c)). However, after the simultaneous inhibition of Bmal1 and Per2, the mRNA expression of Alp, Runx2, and Ocn was much stronger than that in the single-gene suppression groups (Figures $2(\mathrm{a})-2(\mathrm{c})$ ). The difference in the expression of BMAL1, RUNX2, and OCN protein level among the 7 groups was in accordance with the RT-qPCR results (Figures 3(a) $-3(\mathrm{~d})$ ).

After 14 days of osteoinduction, the difference in the mRNA expression of the late osteogenic differentiation marker Ocn among the 7 groups was in accordance with that after 7 days of osteo-induction (Figure 4(a)). The difference in the expression of OCN protein among the 7 groups was in accordance with the RT-qPCR results (Figure 4(b) and 4(c)).

3.3. Alkaline Phosphatase Activity after 7 Days of Osteogenic Induction. After 7 days of osteogenic induction, inhibition of the Bmall or Per2 gene increased the activity of the early osteogenic differentiation marker ALP (Figure 4(d)); after simultaneously inhibiting Bmall and Per2, the activity of ALP was much stronger than that in the single-gene suppression groups.

3.4. Bone Nodule Formation after 14 Days of Osteogenic Induction. After 14 days of osteogenic induction, inhibition of the Bmal1 or Per2 gene increased the formation of calcified nodules (Figure 5). However, after simultaneously inhibiting Bmal1 and Per2, the ability of calcified nodule formation of BMSCs was much stronger than that in the single-gene suppression groups.

\section{Discussion}

The present study showed that the mRNA and protein expression of osteogenic differentiation markers (Alp, 


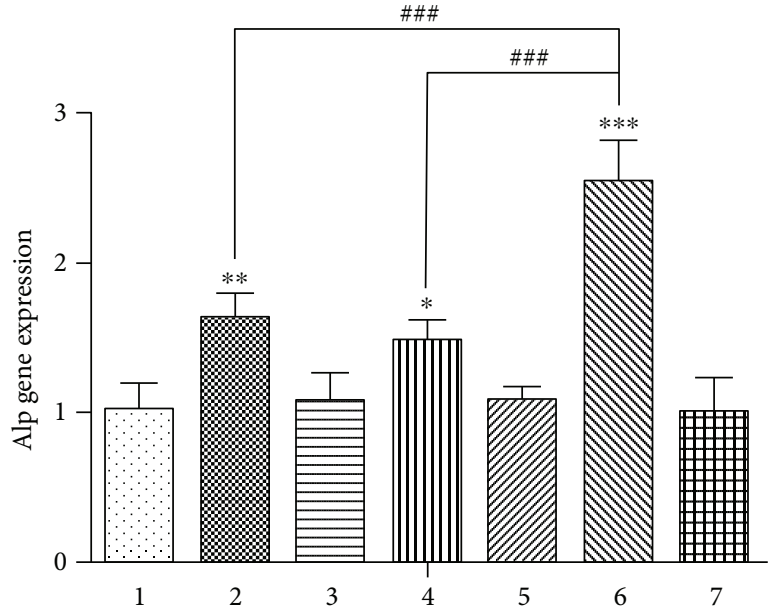

(a)

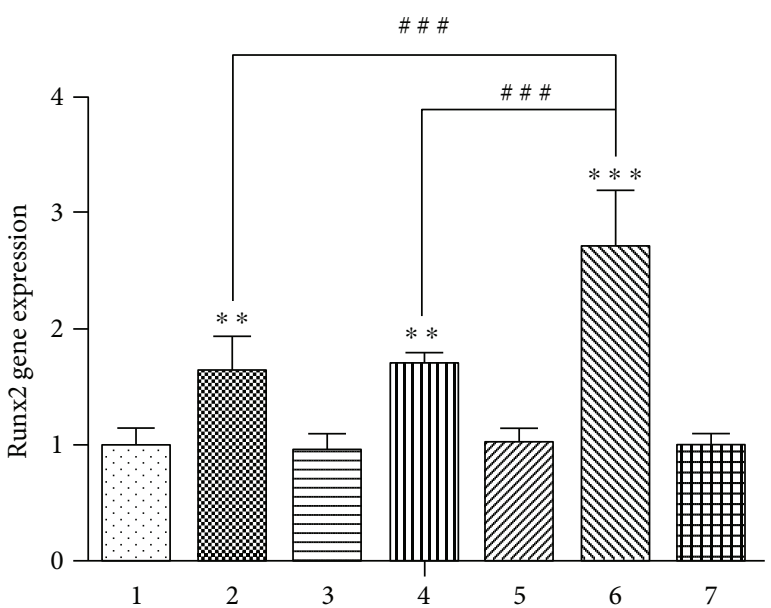

(b)

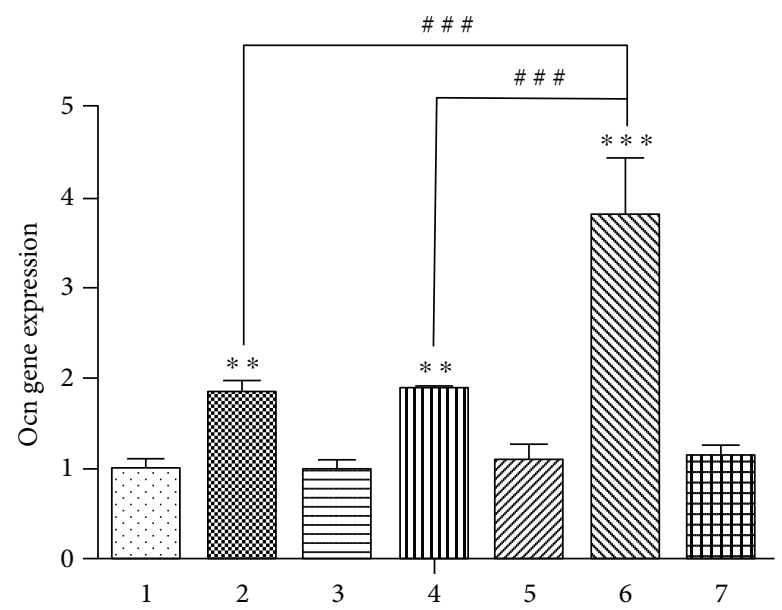

(c)

Figure 2: Alp, Runx2, and Ocn mRNA expression among the groups after 7 days of osteoinduction. (a) Alp mRNA expression. (b) Runx2 mRNA expression. (c) Ocn mRNA expression. Data represent the mean $\pm \operatorname{SD}(n=3)$; the asterisk indicates the group compared to the control groups, that is, group 1 , group 3 , group 5 , or group $7 .\left({ }^{*} p<0.05,{ }^{* *} p<0.01\right.$, and $\left.{ }^{* * *} p<0.001\right)$. The pound key indicates a significant difference between the two groups $\left({ }^{\# \# \#} p<0.001\right)$.

Runx2, and Ocn), the ALP activity, and the ability of calcified nodule formation in BMSCs were enhanced after the inhibition of Bmal1 and Per2, demonstrating that the osteogenic differentiation capability of BMSCs was increased, and Bmal1 and Per2 have negative roles in the regulation of BMSC osteogenic differentiation. After simultaneously inhibiting Bmal1 and Per2, the osteogenic differentiation ability of BMSCs were much stronger than those following singlegene inhibition, indicating that Bmal1 and Per2 may have a synergistic effect on BMSC osteogenic differentiation.

\subsection{The Relationship between Bmal1 and Bone Metabolism.} $\mathrm{Fu}$ et al. demonstrated that Bmal1-/- mice had increased osteoblast numbers and bone-formation parameters, indicating that Bmall had a negative role in the regulation of bone formation [11]. Takarada et al. also showed that in 8-weekold Bmal1-/- mice, the osteoblast surface/bone surface, bone formation rate/bone surface and mineral apposition rate of the femur by histomorphometric analysis increased significantly [13]. Similarly, our data suggest that after the inhibition of Bmall, the osteogenic differentiation capability of BMSCs was enhanced, indicating that Bmall has a negative role in the regulation of bone formation. However, Bmal1-/- mice did not exhibit a high bone mass (HBM), which may be explained by hypogonadism, a condition that increases bone resorption [14-17]. Urinary elimination of deoxypyridinoline crosslinks, a by-product of collagen degradation, was increased in Bmall-l- mice, indicating that bone resorption was indeed enhanced in these mice, explaining, at least in part, the absence of the high bone mass phenotype in Bmal1-/- mice. Fujihara et al. showed that osteoclast-related genes, such as nuclear factor of activated T-cells, cytoplasmic 1 (NFATc1), and cathepsin K (CTSK), showed circadian rhythmicity similar to that of clock genes, such as Bmal1, Per1, and Per2, in the femur (cancellous bone) of mice [7]. A chromatin immunoprecipitation (ChIP) assay revealed the interaction between BMAL1 proteins and the promoter regions of NFATc1 and CTSK. Thus, the circadian clock gene 


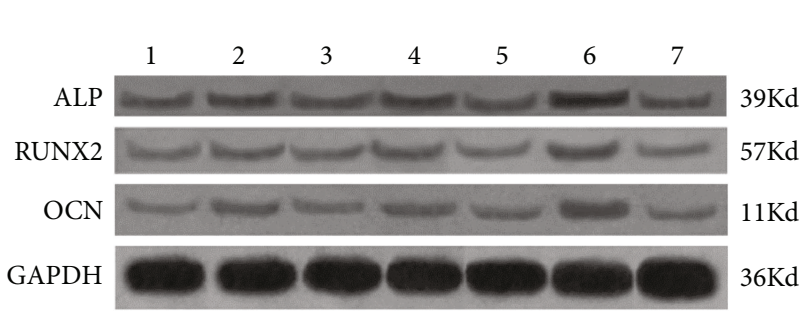

(a)

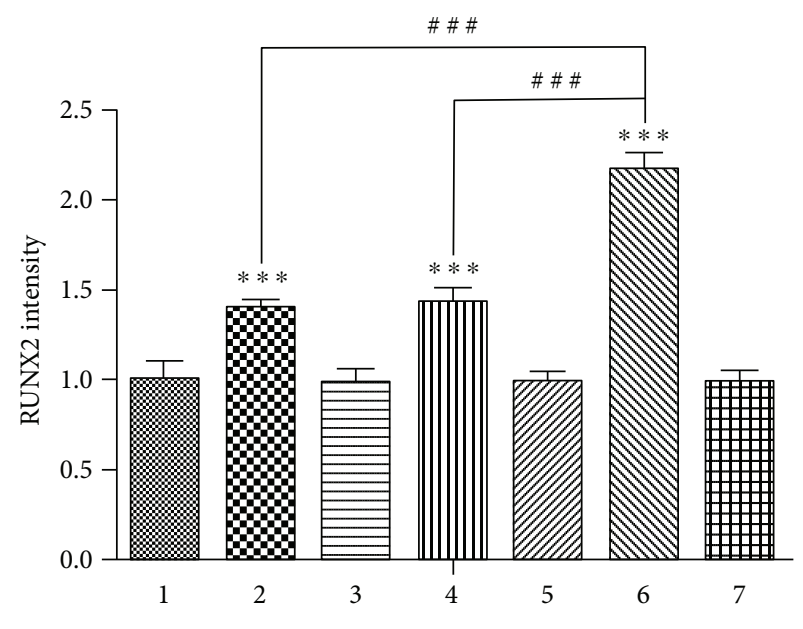

(c)

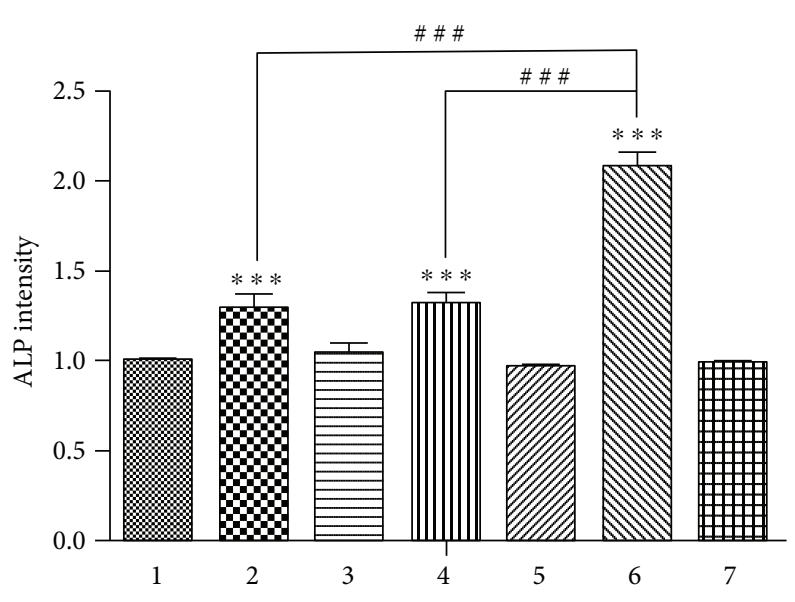

(b)

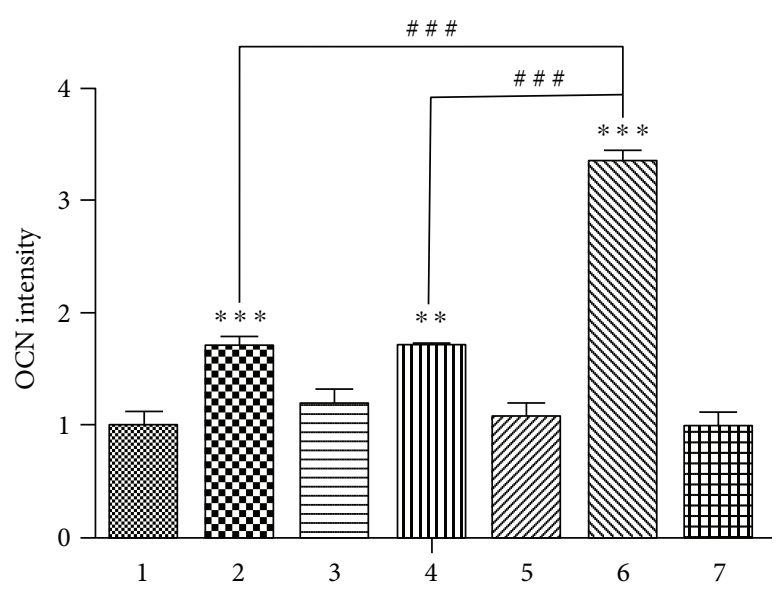

(d)

FIGURE 3: After 7 days of osteoinduction, Western blotting results showed the same trend of ALP, RUNX2, and OCN protein expression as the Bmal1, Runx2, and Ocn mRNA among the seven groups. GAPDH served as the endogenous reference gene. (a) The result of Western blotting. (b) The ALP protein expression intensity. (c) The RUNX2 protein expression intensity. (d) The OCN protein expression intensity. Densitometry was used to quantify the protein level; the protein level was displayed as the ratio of ALP, RUNX2, and OCN relative to GAPDH. Data represent the mean \pm SD $(n=3)$; the asterisk indicates the group compared to the control groups, that is, group 1, group 3, group 5 , or group $7 .{ }^{* *} p<0.01$ and ${ }^{* * *} p<0.001$. The pound key indicates a significant difference between the two groups $\left({ }^{\# \# \#} p<0.001\right.$ ).

Bmall affects not only bone formation metabolism but also bone resorption metabolism.

Samsa et al. found that Bmal1-/- mice had a low bone weight phenotype, exhibiting a decrease in bone mineral density as well as a reduction of the active osteocyte and osteoblast quantity; the ability of differentiation into osteoblasts of BMSCs decreased, which was different from the results of Fu et al. and our results. However, Fu et al. did their experiments mostly on 2-month-old mice, whose skeleton has not reached the peak of bone mass [18]; the mice were still young, were less affected by gene knockout and thus did not display signs of premature aging. In the study of Samsa et al., they did their experiments mostly on 5-month-old mice, when the growth of the skeleton is almost complete; therefore, the mice were more affected by premature aging symptoms caused by gene knockout. Consequently, the low bone mass phenotype of Bmal1-/- mice reported by Samsa et al. was probably because of the premature aging symptoms caused by Bmal1 knockout, which induced bone mass loss, and potential increase in bone formation caused by Bmall knockout were obscured by the impact of premature aging symptominduced bone mass loss.

Our previous study and the research of $\mathrm{Li}$ et al. demonstrated that Bmal1 may regulate bone formation via the Wnt/ $\beta$-catenin pathway and that glycogen synthase kinase$3 \beta$ (GSK-3 $\beta$ ) may act as the medium and link between Bmal1 and the Wnt $/ \beta$-catenin pathway $[19,20]$. Min et al. showed that Bmal1 may promote osteogenic differentiation by regulating the expression of BMP2 in MC3T3-E1 cells [21]. In regard to the relationship between Bmall and bone formation, their results seem to be different from those of this present study. However, the use of models that do not take into account the cellular context, differences in cell preparations or use of nonphysiologic loss- and gain-of-function approaches contributed to the observation of contrasting data. In most cases, there is a lack of environmental signals in vitro experiments, whereas rich environmental signals can be found in vivo. Consequently, for the reliability and 


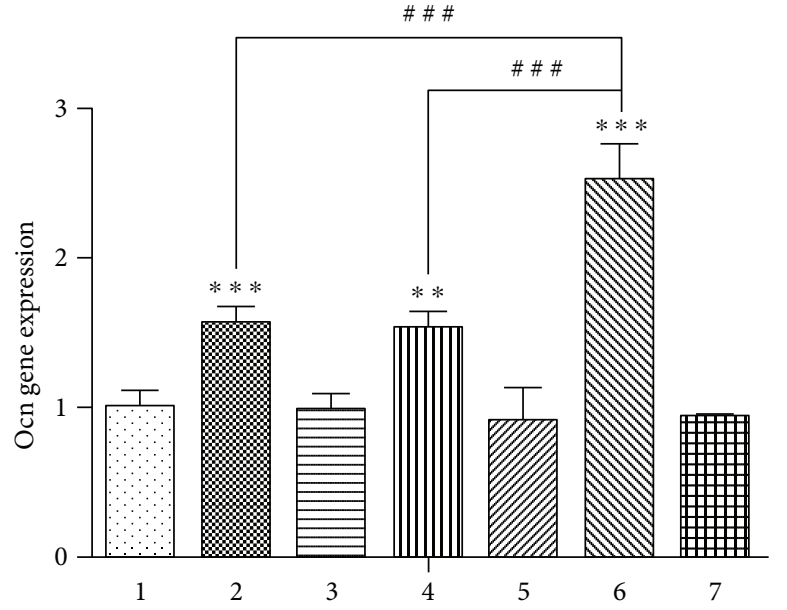

(a)

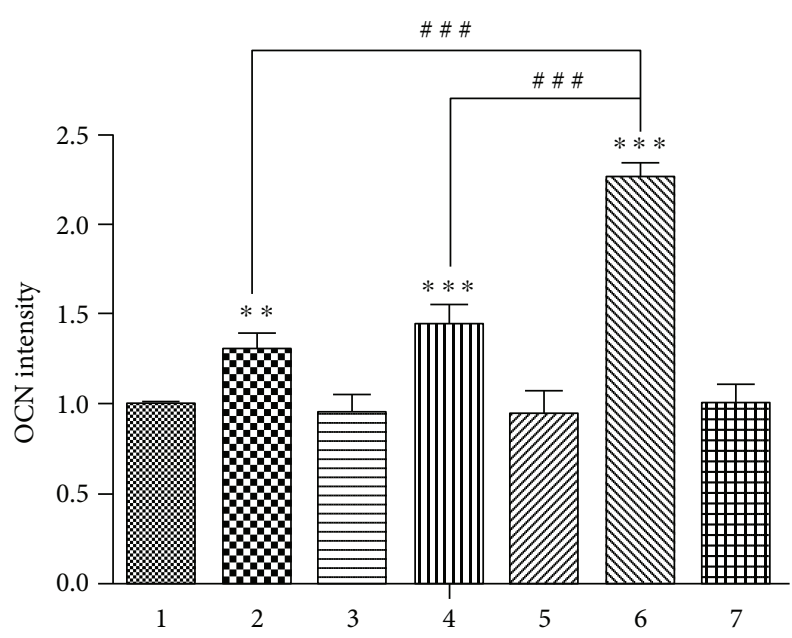

(c)

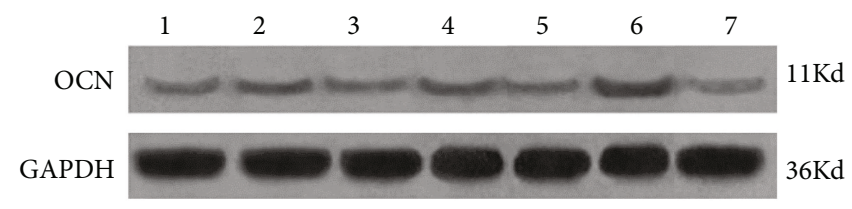

(b)

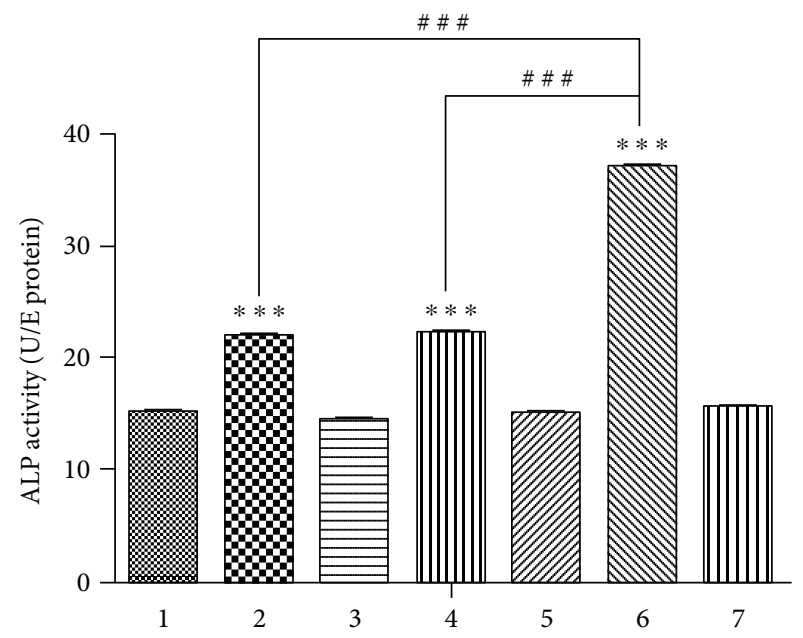

(d)

FIgURE 4: (a) Ocn mRNA expression among the groups after 14 days of osteoinduction. (b) Western blotting results showed the same trend of OCN protein expression as the Ocn mRNA among the seven groups after 14 days of osteoinduction. (c) The OCN protein expression intensity. GAPDH served as the endogenous reference gene. Densitometry was used to quantify the protein level; the protein level was displayed as the ratio of OCN relative to GAPDH. (d) Alkaline phosphatase (ALP) activity among the groups after 7 days of osteoinduction. Data represent the mean $\pm \mathrm{SD}(n=3)$; the asterisk indicates the group compared to the control groups, that is, group 1 , group 3 , group 5 , or group 7 . ${ }^{* *} p<0.01$ and ${ }^{* * *} p<0.001$. The pound key indicates a significant difference between the two groups $\left({ }^{\# \#} p<0.001\right)$.

practicability of the experimental results, we will perform in vivo experiments in the next step.

\subsection{The Relationship between Per2 and Bone Metabolism.} Maronde et al. demonstrated that compared with wild-type mice, the Per $2^{\mathrm{Brdm} 1}$ mutant mice exhibited increased bone formation rate and bone volume at 3,12 , and 48 weeks old. In addition, osteoblast isolation from Per2 ${ }^{\mathrm{Brdm} 1}$ mutant mice had a significantly greater capability to form calcium nodules after 14 days of osteogenic culture [12]. In agreement, our data showed that after inhibition of Per2, the osteogenic differentiation capability of BMSCs was elevated, indicating that Per2 has a negative role in the regulation of bone formation. This present study demonstrated that the ability of osteogenic differentiation in BMSCs was similarly enhanced after the inhibition of the Bmal1 or Per2 gene, whereas Per $2^{\mathrm{Brdm} 1}$ mutant mice displayed a high bone mass phenotype, which was different from the phenotype of Bmal1-/- mice. A possible explanation is that Per2-deficient mice have milder premature aging symptoms than Bmall-deficient mice. And thus, the mice were less affected by the premature aging symptom-induced loss of bone mass. Without the inhibitive effect of Per2 on bone formation, the mice displayed increased bone mass.

$\mathrm{Fu}$ et al. found that Per1-/-; Per2m/m and wild-type mice exhibited similar urinary elimination of deoxypyridinoline crosslinks, indicating that bone resorption was not obviously affected in these mice [11]. Maronde et al. discovered that both Per2 ${ }^{\mathrm{Brdm} 1}$ mutant mice and mice deficient in Cry2 showed obviously incremental bone volume at 12 weeks of age, but Per2 $2^{\mathrm{Brdm} 1}$ mutant mice displayed changes in parameters especial for osteoblasts and displayed no change of the osteoclast activity marker TRAP5b in the circulating serum. However, mice deficient in Cry2 displayed changes 


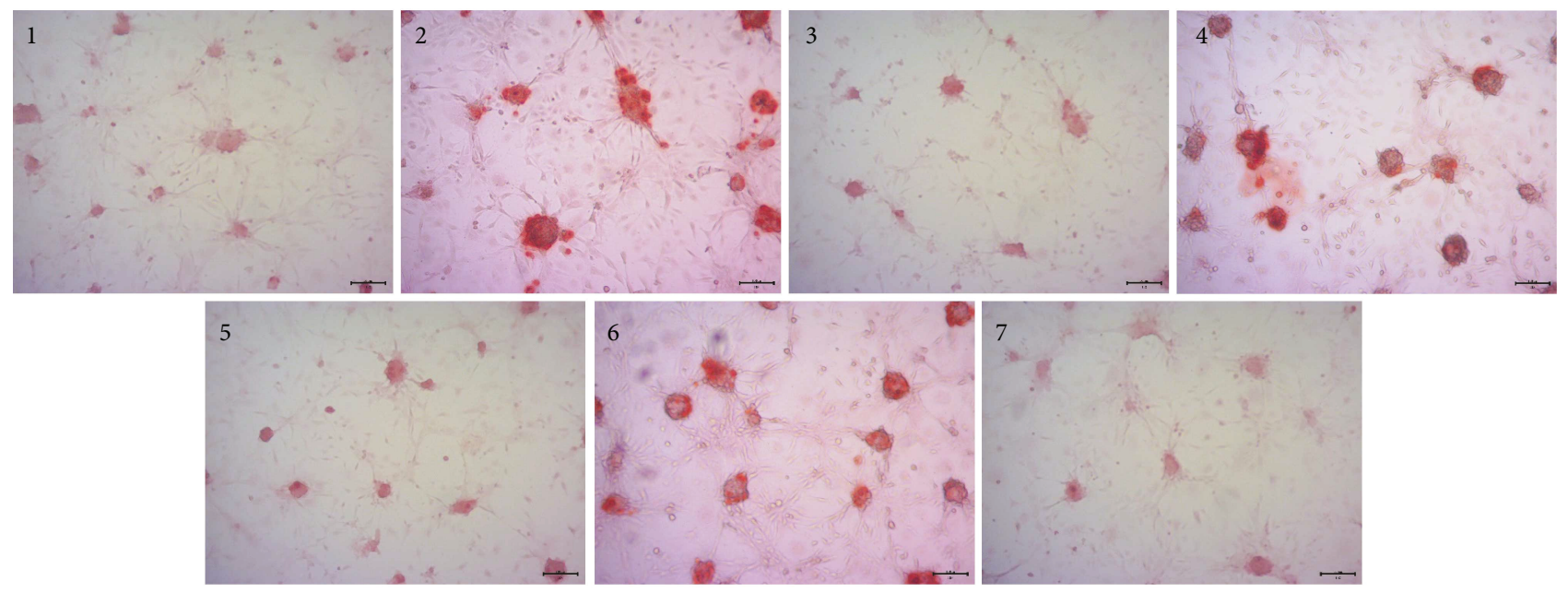

(a)

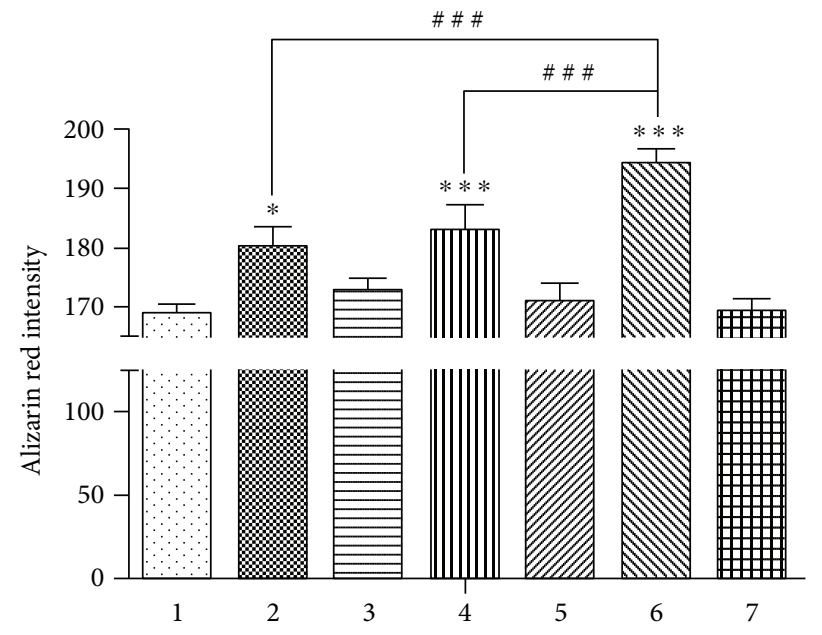

(b)

Figure 5: (a) Bone nodule formation after 14 days of osteogenic induction. The calcified nodules of group 2 and group 4 were more than that of the control groups, that is, group 1, group 3, group 5, or group 7. But the calcified nodule formation of group 6 was better than that of group 2 and group 4. Magnification is 100x. (b) The intensity of Alizarin Red among the groups after 14 days of osteoinduction. Data represent the mean $\pm \mathrm{SD}(n=3)$; the asterisk indicates the group compared to the control groups, that is, group 1 , group 3 , group 5 , or group $7 .{ }^{*} p<0.05$ and ${ }^{* * *} p<0.001$. The pound key indicates a significant difference between the two groups $\left({ }^{\# \#} p<0.001\right)$.

in parameters especial for osteoclasts, which decrease the serum levels of circulating TRAP5b without affecting the osteoblast activity markers. Therefore, Per $2^{\text {Brdm1 }}$ mutant mice increase their bone mass mainly through enhancing bone synthesis, and the circadian gene Per2 affects bone metabolism mainly by regulating bone formation, whereas it does not overtly affect bone resorption.

Regarding the Per2 signaling pathway in the regulation of bone synthesis, studies have found that Per1-/-; Per2m/m, Per1-/-; Per2-/-, and Cry1-/-; Cry2-/- mice all exhibit high bone mass phenotypes, indicating that bone formation is negatively regulated by the molecular clock [11]. Studies found that, while bone-formation parameters and bone mass decreased in wild-type mice after long-term leptin i.c.v. infusion, this treatment consistently enhanced bone-formation parameters in Per1-/-; Per2m/m mice. Their data support that the circadian gene Per mediates the leptin-dependent sympathetic inhibition of bone formation in osteoblasts and that the possible mechanism was via the downregulation of $c-m y c$ expression by Per, which inhibits the expression of G1 cyclin, subsequently extending of the cell cycle and inhibiting osteoblast proliferation.

4.3. The Correlations between Bmal1 and Per2 in the Regulation of Bone Metabolism. The present research showed that Bmal1 and Per2 had negative roles in the regulation of osteogenic differentiation in BMSCs and that Bmal1 and Per2 may have a synergistic effect on BMSC osteogenic differentiation. Several scholars have demonstrated that there is a correlation between Bmal1 and Per2 in terms of their activity and gene expression [22]. The expression of Per1 and Per2 is significantly decreased in all tissues of Bmal1-/- mice, and Bmal1 is also distinctly downregulated in tissues of Per1-/-; Per2-/- mice. In agreement, our data showed that after inhibition of Bmall, the expression of PER2 protein was downregulated. In 
turn, after Per2 was inhibited, the BMAL1 protein expression was also downregulated. It explains, at least in part, the synergistic effect of Bmal1 and Per2 on the osteogenic differentiation of BMSCs.

Output signals from the suprachiasmatic nuclei (SCN) are believed to transmit standard circadian time to peripheral tissues through humoral routes and the sympathetic nervous system $[23,24]$. Some papers have indicated that the sympathetic nervous system mediates the expression of core clock genes and regulates bone remodeling [25]. Hirai et al. demonstrated that isoproterenol (Iso) of MC3T3-E1 osteoblastic cells, a nonselective $\beta$-AR agonist, orchestrated the rhythmic expression of the canonical clock genes Bmal1 and Per2 [26]. Iso also made the mRNA of prostaglandin endoperoxide synthase 2 (Ptgs2, also known as Cox2, an important regulation factor of bone metabolism) express rhythmically. They found that the interaction between Bmal1 and Per2 mediated the rhythmic impacts of Iso on the expression of Ptgs 2 in osteoblasts. In addition, after continuous Iso treatment, Ptgs 2 was significantly decreased in bone. They used a bioinformatics approach to reveal that the promoter of Ptgs2 gene contained E-box and Dbox sequences, demonstrating that the circadian oscillators regulated Ptgs2 gene in osteoblasts. Other studies have shown that the promoter of Per2 contains D- and Ebox-binding elements [27-29]. Their results revealed that $\beta$-AR signaling regulate the expression of Bmall and Per2 in osteoblasts and that the BMAL1 and PER2 proteins control the expression of Ptgs 2 by binding to the E-box of the Ptgs2 promoter, thereby controlling bone metabolism. There is indeed some correlation between Bmal1 and Per2 in bone metabolism regulation, and they can control this complex physiological activity through several shared signaling pathways.

\section{Conclusion}

Our results suggest that the osteogenic differentiation capability of BMSCs is enhanced after the inhibition of Bmal1 or Per2, indicating that Bmall and Per2 have vital negative roles in the regulation of BMSC osteogenic differentiation. Moreover, Bmal1 and Per2 may have a synergistic effect on the osteogenic differentiation of BMSCs.

\section{Conflicts of Interest}

The authors declare that there is no conflict of interest regarding the publication of this article.

\section{Acknowledgments}

This work was supported by a grant from the National Natural Science Foundation of China (no. 81371113).

\section{References}

[1] C. S. Soltanoff, S. Yang, W. Chen, and Y. P. Li, "Signaling networks that control the lineage commitment and differentiation of bone cells," Critical Reviews ${ }^{\mathrm{T} M}$ in Eukaryotic Gene Expression, vol. 19, no. 1, pp. 1-46, 2009.
[2] D. Feskanich, S. E. Hankinson, and E. S. Schernhammer, "Nightshift work and fracture risk: the Nurses' Health Study," Osteoporosis International, vol. 20, no. 4, pp. 537-542, 2009.

[3] I. Quevedo and A. M. Zuniga, "Low bone mineral density in rotating-shift workers," Journal of Clinical Densitometry, vol. 13, no. 4, pp. 467-469, 2010.

[4] M. Dudek and Q. J. Meng, "Running on time: the role of circadian clocks in the musculoskeletal system," Biochemical Journal, vol. 463, no. 1, pp. 1-8, 2014.

[5] A. R. Guntur, M. Kawai, P. Le et al., "An essential role for the circadian-regulated gene nocturnin in osteogenesis: the importance of local timekeeping in skeletal homeostasis," Annals of the New York Academy of Sciences, vol. 1237, no. 1, pp. 5863, 2011.

[6] J.-D. P. McElderry, G. Zhao, A. Khmaladze, C. G. Wilson, R. T. Franceschi, and M. D. Morris, "Tracking circadian rhythms of bone mineral deposition in murine calvarial organ cultures," Journal of Bone and Mineral Research, vol. 28, no. 8, pp. 1846-1854, 2013.

[7] Y. Fujihara, H. Kondo, T. Noguchi, and A. Togari, "Glucocorticoids mediate circadian timing in peripheral osteoclasts resulting in the circadian expression rhythm of osteoclastrelated genes," Bone, vol. 61, pp. 1-9, 2014.

[8] M. K. Bunger, J. A. Walisser, R. Sullivan et al., "Progressive arthropathy in mice with a targeted disruption of the Mop3/ Bmal-1 locus," Genesis, vol. 41, no. 3, pp. 122-132, 2005.

[9] R. V. Kondratov, A. A. Kondratova, V. Y. Gorbacheva, O. V. Vykhovanets, and M. P. Antoch, "Early aging and agerelated pathologies in mice deficient in BMAL1, the core componentof the circadian clock," Genes \& Development, vol. 20, no. 14, pp. 1868-1873, 2006.

[10] W. E. Samsa, A. Vasanji, R. J. Midura, and R. V. Kondratov, "Deficiency of circadian clock protein BMAL1 in mice results in a low bone mass phenotype," Bone, vol. 84, pp. 194-203, 2016.

[11] L. Fu, M. S. Patel, A. Bradley, E. F. Wagner, and G. Karsenty, "The molecular clock mediates leptin-regulated bone formation," Cell, vol. 122, no. 5, pp. 803-815, 2005.

[12] E. Maronde, A. F. Schilling, S. Seitz et al., "The clock genes Period 2 and Cryptochrome 2 differentially balance bone formation," PLoS One, vol. 5, no. 7, article e11527, 2010.

[13] T. Takarada, C. Xu, H. Ochi et al., "Bone resorption is regulated by circadian clock in osteoblasts," Journal of Bone and Mineral Research, vol. 32, no. 4, pp. 872-881, 2017.

[14] J. S. Finkelstein, H. Lee, B. Z. Leder et al., "Gonadal steroiddependent effects on bone turnover and bone mineral density in men," Journal of Clinical Investigation, vol. 126, no. 3, pp. 1114-1125, 2016.

[15] J. S. Finkelstein, S. E. Brockwell, V. Mehta et al., "Bone mineral density changes during the menopause transition in a multiethnic cohort of women," The Journal of Clinical Endocrinology \& Metabolism, vol. 93, no. 3, pp. 861-868, 2008.

[16] S. L. Greenspan, P. Coates, S. M. Sereika, J. B. Nelson, D. L. Trump, and N. M. Resnick, "Bone loss after initiation of androgen deprivation therapy in patients with prostate cancer," The Journal of Clinical Endocrinology \& Metabolism, vol. 90, no. 12, pp. 6410-6417, 2005.

[17] M. R. Smith, F. J. McGovern, A. L. Zietman et al., "Pamidronate to prevent bone loss during androgen-deprivation therapy for prostate cancer," New England Journal of Medicine, vol. 345 , no. 13 , pp. 948-955, 2001. 
[18] R. L. Jilka, "The relevance of mouse models for investigating age-related bone loss in humans," The Journals of Gerontology Series A: Biological Sciences and Medical Sciences, vol. 68, no. 10, pp. 1209-1217, 2013.

[19] Y. He, Y. Chen, Q. Zhao, and Z. Tan, "Roles of brain and muscle ARNT-like 1 and Wnt antagonist Dkk1 during osteogenesis of bone marrow stromal cells," Cell Proliferation, vol. 46, no. 6, pp. 644-653, 2013.

[20] X. Li, N. Liu, Y. Wang et al., "Brain and muscle aryl hydrocarbon receptor nuclear translocator-like protein-1 cooperates with glycogen synthase kinase- $3 \beta$ to regulate osteogenesis of bone-marrow mesenchymal stem cells in type 2 diabetes," Molecular and Cellular Endocrinology, vol. 440, pp. 93-105, 2017.

[21] H. Y. Min, K. M. Kim, G. Wee, E. J. Kim, and W. G. Jang, "Bmal1 induces osteoblast differentiation via regulation of BMP2 expression in MC3T3-E1 cells," Life Sciences, vol. 162, pp. 41-46, 2016.

[22] R. V. Kondratov, "A role of the circadian system and circadian proteins in aging," Ageing Research Reviews, vol. 6, no. 1, pp. 12-27, 2007.

[23] S. Komoto, H. Kondo, O. Fukuta, and A. Togari, "Comparison of $\beta$-adrenergic and glucocorticoid signaling on clock gene and osteoblast-related gene expressions in human osteoblast," Chronobiology International, vol. 29, no. 1, pp. 66-74, 2012.

[24] C. J. Thomas, T. P. Cleland, S. Zhang, C. M. Gundberg, and D. Vashishth, "Identification and characterization of glycation adducts on osteocalcin," Analytical Biochemistry, vol. 525, pp. 46-53, 2017.

[25] H. Kondo and A. Togari, "Continuous treatment with a lowdose $\beta$-agonist reduces bone mass by increasing bone resorption without suppressing bone formation," Calcified Tissue International, vol. 88, no. 1, pp. 23-32, 2011.

[26] T. Hirai, K. Tanaka, and A. Togari, “ $\beta$-adrenergic receptor signaling regulates Ptgs 2 by driving circadian gene expression in osteoblasts," Journal of Cell Science, vol. 127, no. 17, pp. 3711-3719, 2014.

[27] K. Yagita and H. Okamura, "Forskolin induces circadian gene expression of $r$ Per1, $r P e r 2$ and $d b p$ in mammalian rat-1 fibroblasts," FEBS Letters, vol. 465, no. 1, pp. 79-82, 2000.

[28] S. H. Yoo, C. H. Ko, P. L. Lowrey et al., "A noncanonical E-box enhancer drives mouse Period2 circadian oscillations in vivo," Proceedings of the National Academy of Sciences of the United States of America, vol. 102, no. 7, pp. 2608-2613, 2005.

[29] T. Ohno, Y. Onishi, and N. Ishida, "A novel E4BP4 element drives circadian expression of mPeriod2," Nucleic Acids Research, vol. 35, no. 2, pp. 648-655, 2007. 


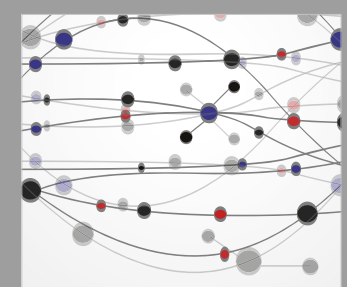

The Scientific World Journal
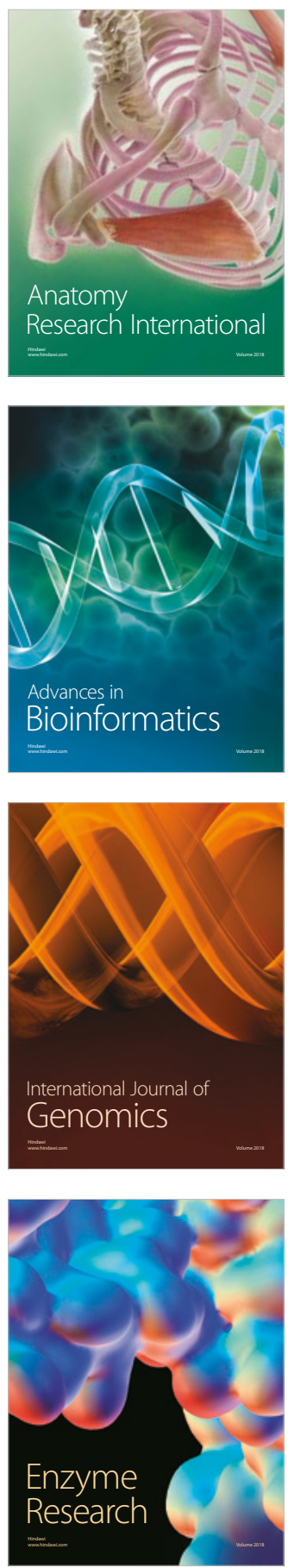
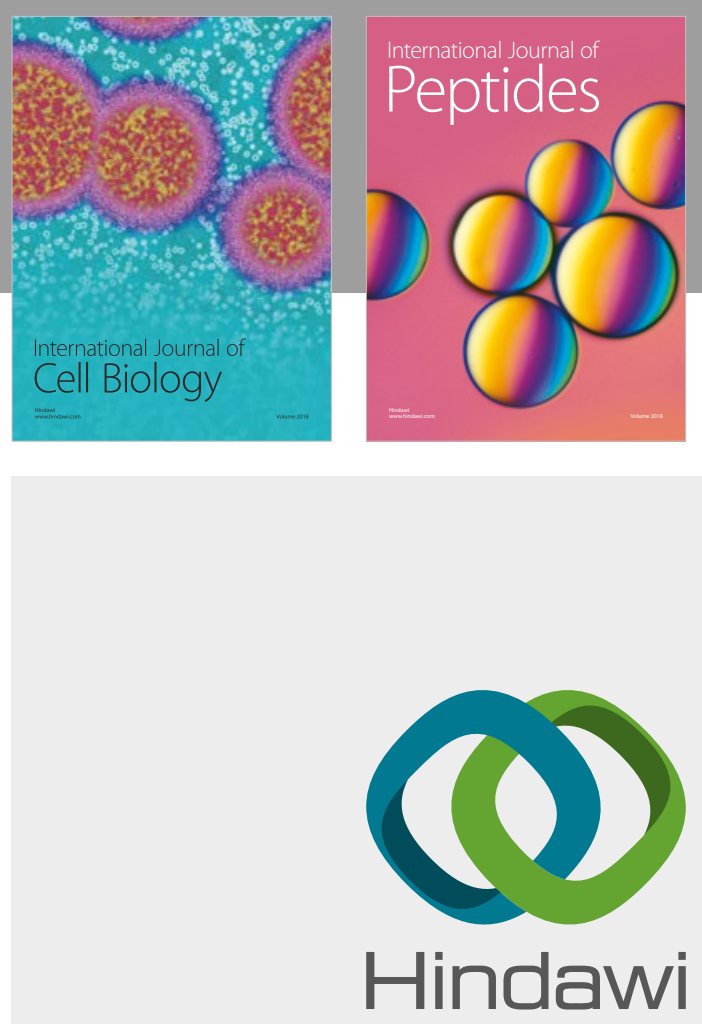

Submit your manuscripts at

www.hindawi.com
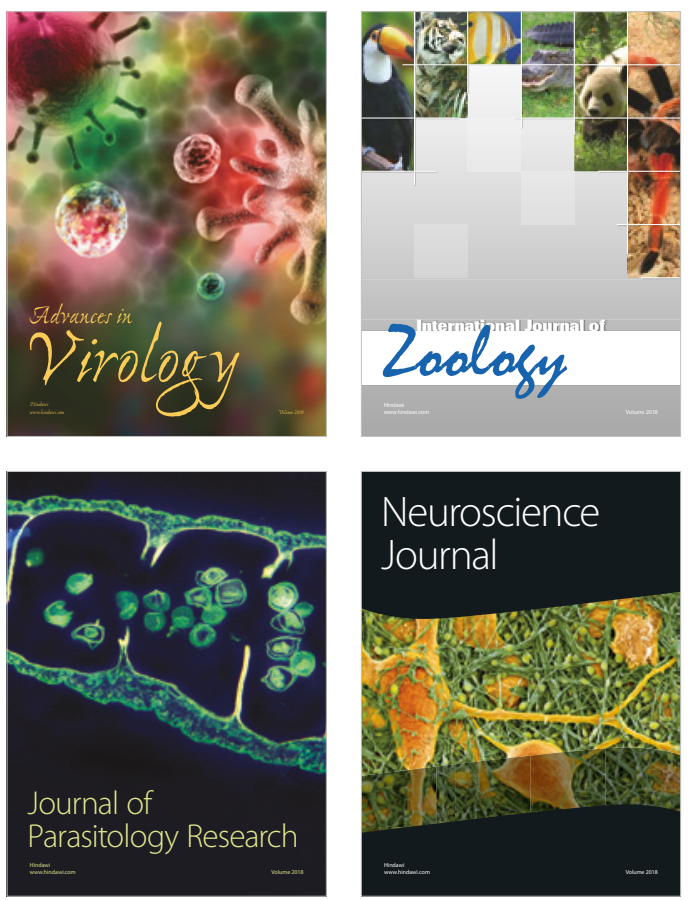
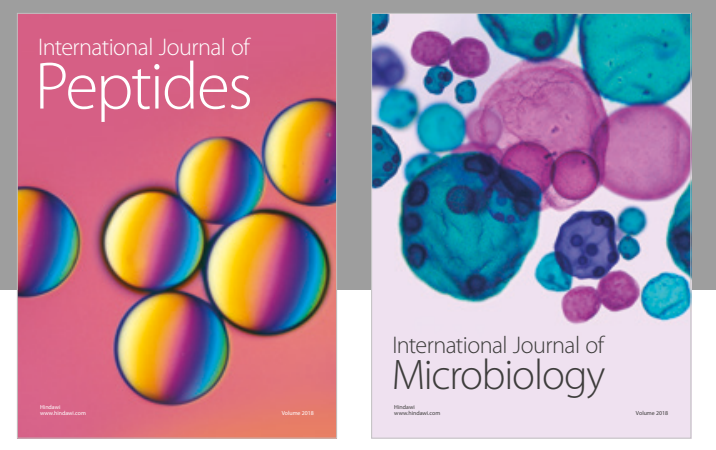

nternational Journal of Microbiology
Journal of
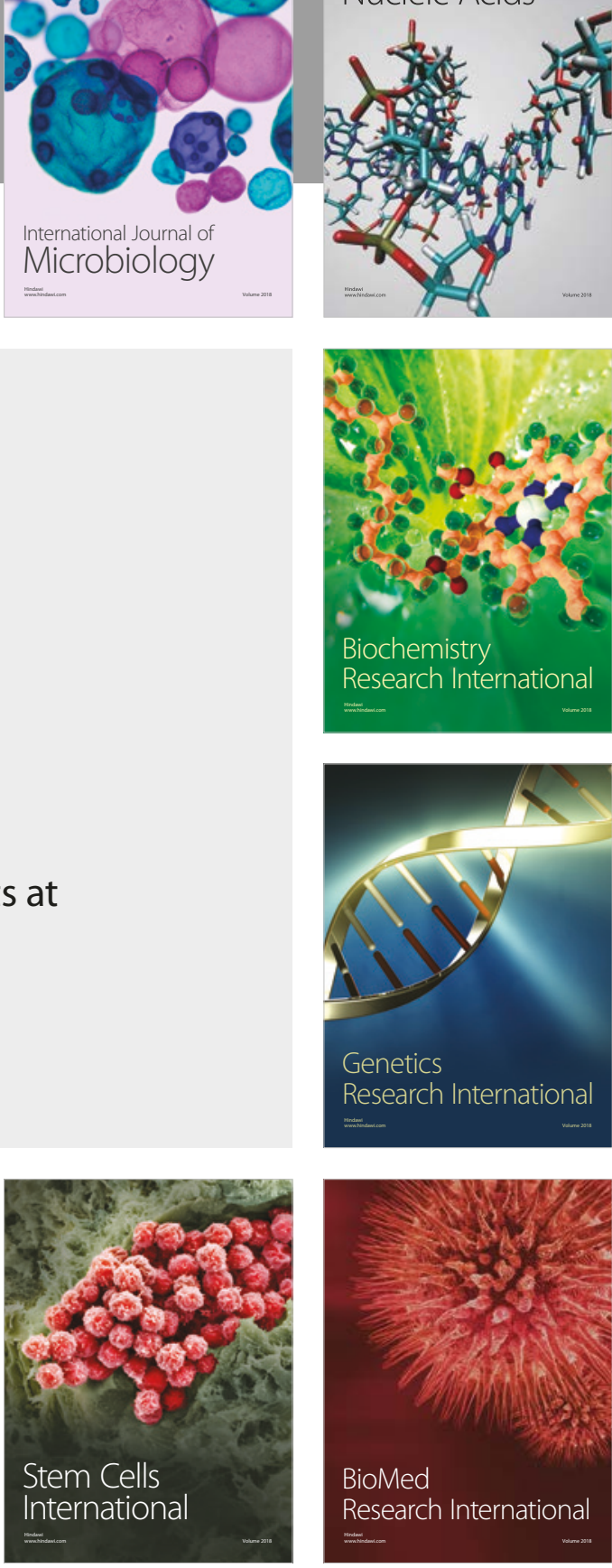
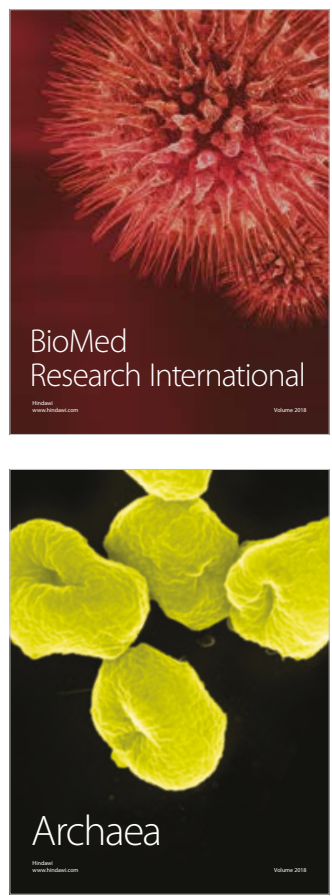